\title{
Testing Optimized Principal Component Analysis on Coronagraphic Images of the Fomalhaut System
}

\author{
Tiffany Meshkat ${ }^{1}$, Matthew Kenworthy ${ }^{1}$, Sascha P. Quanz ${ }^{2}$ \\ and Adam Amara ${ }^{2}$ \\ ${ }^{1}$ Sterrewacht Leiden, \\ P.O. Box 9513, Niels Bohrweg 2, 2300 RA Leiden, The Netherlands \\ email: meshkat@strw.leidenuniv.nl \\ ${ }^{2}$ Institute for Astronomy, ETH Zurich, \\ Wolfgang-Pauli-Strasse 27, 8093 Zurich, Switzerland
}

\begin{abstract}
We present the results of a study to optimize the principal component analysis (PCA) algorithm for planet detection, a new algorithm complementing ADI and LOCI for increasing the contrast achievable next to a bright star. We apply PCA to our Fomalhaut VLT NACO Apodizing Phase Plate NB4.05 data.
\end{abstract}

Keywords. planets and satellites: detection - data analysis techniques: image processing, PCA, LOCI - stars: individual (Fomalhaut)

\section{Data}

Data were obtained at the VLT/UT4 with NaCo (Lenzen et al. 2003, Rousset et al. 2003) in July and August 2011 (087.C-0701(B)). The Fomalhaut data were first analyzed and published in Kenworthy et al. 2013. Fomalhaut, a young nearby A4V star, is surrounded by an inclined dust debris belt with a planet that has been imaged in the optical, 119 AU away from the star (Kalas et al. 2008). Kenworthy et al. 2013 searched for planets close in around Fomalhaut, from 4-10 AU, using the LOCI algorithm.

Fomalhaut was used as the natural guide star with the visible band wavefront sensor. The L27 camera on NaCo was used with the NB4.05 filter $(\lambda=4.051 \mu \mathrm{m}$ and $\Delta \lambda=$ $0.02 \mu \mathrm{m}$ ) and the Apodizing Phase Plate coronagraph (APP, Kenworthy et al. 2010, Quanz et al. 2010) to provide additional diffraction suppression. We used pupil tracking mode to perform ADI (Marois et al. 2006). The PSF core is intentionally saturated to increase the signal from any potential companions.

The unsaturated data is used to calibrate the photometry in the saturated images. Fake planets are subsequently used to determine the limiting magnitude after image processing with LOCI, PCA, and variations on PCA in this work.

\section{Results}

Optimized PCA yields a more sensitive contrast curve (up to 1 magnitude gain) than the LOCI algorithm at small inner working angles for our Fomalhaut dataset (Figure 1). See Meshkat et al. (2013, submitted), for more details on this work. 


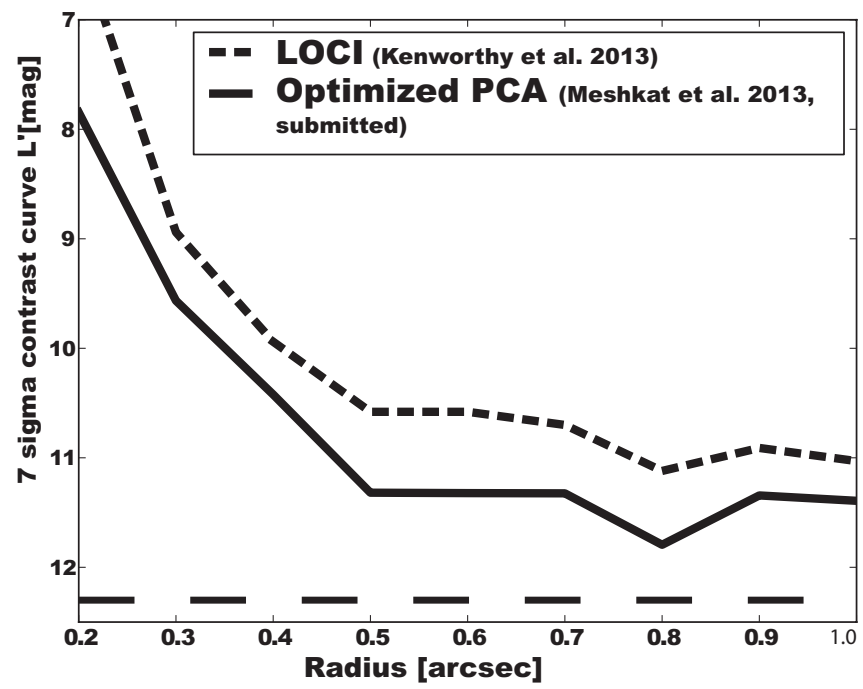

Figure 1. Contrast curves for a $7 \sigma$ detection of a point source in our Fomalhaut APP data processed with LOCI and PCA. The LOCI dashed curve is adapted from Kenworthy et al. 2013 to a $7 \sigma$ detection. The PCA solid contrast curve is the mean value for artificial planets inserted at two PAs on opposite sides of the star $\left(\mathrm{PA}=45^{\circ}\right.$ and $\left.225^{\circ}\right)$. The wide dashed straight line is the background limit.

\section{References}

Kalas, P., Graham, J. R., Chiang, E., Fitzgerald, M. P., Clampin, M., Kite, E. S., Stapelfeldt, K., Marois, C., \& Krist, J. 2013, Sci, 322, 1345K

Kenworthy, M. A., Meshkat, T., Quanz, S. P., Girard, J. H., Meyer, M. R., \& Kasper, M. 2013, $A p J, 764,7 \mathrm{~K}$

Kenworthy, M. A., Quanz, S. P., Meyer, M. R., Kasper, M. E., Lenzen, R., Codona, J. L., Girard, J. H., \& Hinz, P. M. 2010, SPIE, 7735E, 103K

Lenzen, R., Hartung, M., Brandner, W., Finger, G., Hubin, N. N., Lacombe, F., Lagrange, A.-M., Lehnert, M. D., Moorwood, A. F. M., \& Mouillet, D. 2003, SPIE, 4841, 944L

Marois, C., Lafrenière, D., Doyon, R., Macintosh, B., \& Nadeau, D. 2006, ApJ, 641, 556M

Rousset, G., Lacombe, F., Puget, P., Hubin, N. N., Gendron, E., Fusco, T., Arsenault, R., Charton, J., Feautrier, P., Gigan, P., Kern, P. Y., Lagrange, A.-M., Madec, P.-Y., Mouillet, D., Rabaud, D., Rabou, P., Stadler, E., \& Zins, G. 2003, SPIE, 4839, 140R

Quanz, S. P., Meyer, M. R., Kenworthy, M. A., Girard, J. H. V., Kasper, M., Lagrange, A.-M., Apai, D., Boccaletti, A., Bonnefoy, M., Chauvin, G., Hinz, P. M., \& Lenzen, R. 2010, ApJ, $722 \mathrm{~L}, 49 \mathrm{Q}$ 